\title{
Nachhaltigkeit und Unternehmen
}

\section{Thesen}

\section{Rudi Kurz · Werner Wild · BUND-Arbeitskreis Wirtschaft und Finanzen}

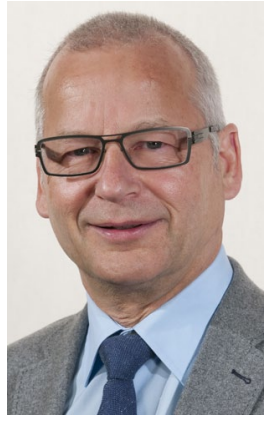

Rudi Kurz

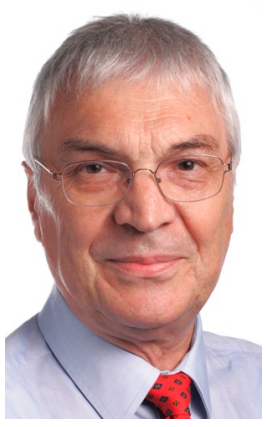

Werner Wild

\section{Vorbemerkung: Unternehmen sind zentrale Akteure für Nachhaltige Entwicklung (NE)}

Nachhaltige Entwicklung ist ohne den Beitrag von Unternehmen nicht möglich. Unternehmen haben einen erheblichen Einfluss auf die sozialen und ökologischen Bedingungen, vor allem durch die von ihnen verantworteten Produktionsbedingungen, den Produktionsverfahren, die Produkte und Dienstleistungen, aber auch durch ihre Mitwirkung an der politischen Willensbildung. In Unternehmen wird über Res-

R. Kurz $(\bowtie) \cdot$ W. Wild $\cdot$ BUND-Arbeitskreis

Wirtschaft und Finanzen

Arbeitskreis Wirtschaft und Finanzen,

Bund für Umwelt und Naturschutz Deutschland (BUND),

Friends of the Earth Germany,

Berlin, Deutschland

E-Mail: rudi.kurz@hs-pforzheim.de

W. Wild

E-Mail: werner.wild@th-nuernberg.de

sourcenverbrauch und Emissionen ebenso entschieden wie über Arbeitsplätze und Arbeitsbedingungen. Multinationale Konzerne treiben die Globalisierung der Wirtschaft voran. Sie intensivieren die internationale Arbeitsteilung, indem sie Produktionsstätten auf der ganzen Welt errichten, mit Zulieferbetrieben in einer weltumspannenden Wertschöpfungskette kooperieren, mit Produkten auf dem globalen Markt handeln - und dies oftmals unter Ausnutzung geringer oder fehlender Rechte der Menschen und durch Raubbau an natürlichen Ressourcen.

Viele Unternehmen haben diese Probleme inzwischen verstanden und als neue, zusätzliche Herausforderung für unternehmerisches Handeln begriffen. Allerdings sind es immer noch (zu) wenige Unternehmen, die adäquate Antworten auf die Herausforderung gefunden haben. Unternehmerische und damit gesellschaftliche Verbesserungspotenziale bleiben ungenutzt. Unternehmerische Aktivitäten im Bereich Nachhaltigkeit konzentrieren sich auf die Bereiche Marketing und Öffentlichkeitsarbeit. Im Mittelpunkt stehen Nachhaltigkeitsrhetorik und schön gestaltete Nachhaltigkeitsberichte. Die Frage aber muss lauten, welchen Beitrag Unternehmen mit ihrem Kerngeschäft zu einer nachhaltigen Entwicklung leisten? Wie können sie ihr Geschäftsmodell neu ausrichten, um soziale und ökologische Verantwortung im normativen, strategischen und operativen Handeln wahrzunehmen?

Die folgenden Thesen sollen eine kritische Bestandsaufnahme zu unternehmerischer Nachhaltigkeit, vor allem aber eine Aufforderung zu wirksamerer Wahrnehmung der gesellschaftlichen Verantwortung von Unternehmen sein. Wenn hier pauschal von „Unternehmen“ die Rede ist, soll damit nicht verkannt werden, dass es erhebliche Unterschiede zwischen Unternehmen gibt, z. B. in der Unternehmensgröße, der Eigentümerstruktur, der Rechtsform, der Branche. Solche Differenzierungen sind hier nicht möglich, 
sollten aber in der weiteren Diskussion Berücksichtigung finden.

\subsection{Unternehmen sind strukturpolitische Akteure -} leisten aber wenig zur Umsetzung des Leitbilds einer nachhaltigen Entwicklung

Unternehmen leisten zu wenig Unterstützung für die Reform der Rahmenbedingungen - und sind daher mitverantwortlich dafür, dass Nachhaltiges Wirtschaften nicht das optimale Umfeld (für Pioniere) hat. Konkret: Abbau umweltschädlicher Subventionen, Fortsetzung und Weiterentwicklung der Ökologischen Steuerreform, Festlegung strenger Grenzwerte, z. B. für die Automobilbranche, menschen- und familiengerechte Arbeitsformen und - zeiten.

Unternehmen wirken auf ihr gesamtes Umfeld ein und gestalten dieses aktiv mit. ${ }^{1}$ Sie wirken insbesondere bei der Formulierung staatlicher Rahmensetzungen mit und beeinflussen so die Leitlinien und Handlungsfelder staatlicher Politik. Unternehmen und ihre Verbände verweigern einer verbindlichen Rechenschaftspflicht ihre Zustimmung, sie setzen demgegenüber auf Selbstverpflichtungen und Freiwilligkeit, verhindern einen wirksamen Emissionshandel und engagieren sich nicht für strukturelle Veränderungen zugunsten einer nachhaltigen Finanzpolitik. Angesichts der globalen nicht-nachhaltigen Entwicklungen, insbesondere bei Klimaschutz und Biodiversität, ist ein verstärktes Engagement auch der Unternehmen für veränderte Rahmenbedingungen dringlicher denn je. Die aktuelle Flüchtlingsproblematik könnte sich erheblich verschärfen, wenn sich der Klimawandel ungebremst fortsetzt und neue Flüchtlingsströme induziert. Als Orientierungsrahmen für unternehmerisches Handeln können die UN Sustainable Development Goals (Agenda 2030) dienen, die auch in die Weiterentwicklung der deutschen Nationalen Nachhaltigkeitsstrategie einfließen sollen.

\subsection{Die meisten Unternehmen betreiben vor allem Nachhaltigkeitsrhetorik}

Unternehmen haben die Herausforderung der nachhaltigen Entwicklung noch nicht wirklich angenommen.

\footnotetext{
${ }^{1}$ Vgl. Uwe Schneidewind, Die Unternehmung als strukturpolitischer Akteur, Marburg 1998. Die Rolle von Unternehmen lässt sich normativ beschreiben, indem sie aufgefordert werden, ,sich für veränderte Rahmenbedingungen einzusetzen und sich in die gesellschaftliche und politische Debatte einzumischen, um die Transformation zu einer Postwachstumsgesellschaft zu unterstützen und so die natürlichen Lebensgrundlagen zu erhalten und die sozialen Unterschiede zu verringern“. (I. Seidl/A. Zahrnt, Strukturwandel fordert Neuausrichtung von Geschäftsmodelle. In: Ökologisches Wirtschaften Heft $1 / 2013$, S. 29.).
}

Es dominieren Nachhaltigkeits-Rhetorik, -Aktionismus und Schaufenster(-Best-Practice-)Beispiele. Es mangelt an der strategischen Verankerung in Form von Key Principles und an empirisch belegten, signifikanten Verbesserungen.

In Studien zum Nachhaltigkeitsengagement von Unternehmen wird festgestellt, dass Nachhaltigkeit im alltäglichen Geschäft, aber auch in zentralen Sachfunktionen weitgehend nebenher läuft. Die größte Motivation, Nachhaltigkeitsthemen zu bearbeiten, ist für deutsche Unternehmen die Pflege ihrer Reputation und ihres Images. Nur knapp die Hälfte der befragten Unternehmen analysiert überhaupt die Auswirkungen auf Produktivität, Effizienz und Effektivität. ${ }^{2}$

Das Nachhaltigkeits-Management ist bei einem Großteil der Unternehmen noch in der Findungsphase. In mehr als der Hälfte der Firmen klafft eine beträchtliche Lücke zwischen selbst definiertem Anspruch und den aktuellen organisatorischen Strukturen. Mittelfristig ist das insbesondere nach außen eine erhebliche Gefahr für die Glaubwürdigkeit des Unternehmens und damit auch ein wirtschaftliches Risiko. ${ }^{3}$ Ein aktuelles Beispiel für „Greenwashing“" offenbart der Volkswagen-Konzern, der u.a. den UN Global Compact unterzeichnet hat und seit Jahren Umwelt- und Nachhaltigkeitsberichte veröffentlicht. Die im September 2015 öffentlich gewordenen Manipulationen des VWKonzerns bei der Messung der Abgase von Dieselfahrzeugen sind ein Beleg für die schönfärberische Rhetorik des Unternehmens, für die die PR-Abteilung zuständig war. Offensichtlich fehlt es im Konzern an einer strategischen Orientierung zur Umsetzung der Ziele einer nachhaltigen Entwicklung in der Kultur, der Politik und der Strategie des Unternehmens.

\subsection{Nachhaltigkeit erscheint in der \\ Unternehmenskommunikation als beliebig einsetzbarer Begriff}

Unternehmen tragen zur Verwässerung des Nachhaltigkeitsbegriffs bei, nehmen wenig Bezug auf konkrete gesellschaftliche Nachhaltigkeitsziele, insbesondere Zielsetzungen im Klimaschutz $\left(\mathrm{CO}_{2}\right.$-Emissionen bis

\footnotetext{
${ }^{2}$ Vgl. Corporate Sustainability Barometer der Leuphana-Universität im Auftrag von PricewaterhouseCoopers. Verfügbar unter: http:// www.pwc.de/de/nachhaltigkeit/nachhaltigkeit-laeuft-noch-immernebenher.jhtml; Vgl. V.Stahlmann/J. Clausen, Umweltleistung von Unternehmen. Von der Öko-Effizienz zur Öko-Effektivität, Wiesbaden 2000.

${ }^{3}$ Alexander v. Preen, Geschäftsführer der Managementberatung Kienbaum. http://www.business-wissen.de/unternehmensfuehrung/nachhaltigkeits-management-unternehmen-arbeiten-wenig-nachhaltig.
} 
2050 um $80 \%$ reduzieren), Biodiversitäts- und Flächenschutzziele, Material-/Rohstoff- und Energieeffizienz. Jedem, der den Begriff „Nachhaltigkeit“ benutzen will, sollte klar sein, dass er sich auf eine Diskussion über ökologische und soziale Grenzen unternehmerischer Tätigkeit einlässt.

Unternehmen tragen zu wenig proaktiv zur Debatte um die Bildung verpflichtender Ziele bei und setzen sich auch nicht in ihren jeweiligen Nachhaltigkeitsberichten mit den gesellschaftlichen Zielen auseinander. Zum Beispiel die Nachhaltigkeitsziele, auf die sich die Gesellschaft verständigt hat ${ }^{4}$, werden nicht auf die Ebene der Unternehmen herunter gebrochen und sind keineswegs handlungsleitend für unternehmerische Entscheidungen. Ein Emissionsbudget für ein Unternehmen könnte durch das Verhältnis von Bruttowertschöpfung des jeweiligen Unternehmens zur globalen Bruttowertschöpfung ermittelt werden.

Die ökologischen Auswirkungen der Geschäftstätigkeit eines Unternehmens sind auf die gesellschaftlichen Nachhaltigkeitsziele zu beziehen. Es geht darum festzustellen, ,,inwiefern ein Unternehmen mit seinen Produkten innerhalb oder außerhalb ökologischer Grenzen des Wachstums operiert". 5.

\subsection{Unzureichende Kenntnis in Unternehmen über die von} ihrer Geschäftstätigkeit verursachten ökologischen und sozialen Auswirkungen

Das betriebliche Rechnungswesen ist genauso verzerrt wie das volkswirtschaftliche: Periodenergebnisse (Output, Umsatz, Gewinn) stehen im Vordergrund, Substanzerhaltung bei den Vermögenswerten wird vernachlässigt (Humankapital, Sozialkapital, Naturkapital).

In den Abteilungen Rechnungswesen/Controlling wurden bislang die sozialen und ökologischen Herausforderungen des Unternehmens kaum beachtet oder vollkommen ignoriert. Diese Sachfunktionen üben aber wesentlichen Einfluss auf die strategische Ausrichtung, Steuerung und Gestaltung des Unternehmens aus. Erst allmählich wird im betrieblichen Rechnungswesen die ökonomische Relevanz nachhaltigen Handelns wahrgenommen. Soziale und ökologische Risiken verursachen Kosten und mit wachsender ökonomischer Bedeutung dieser Kosten (z. B. Bumerangeffekte, Reputationsverluste, Boykotte, staatliche Defensivkosten) wird die Perspektive der Substanzerhaltung notwendig für das Rechnungswesen. ${ }^{6}$

\footnotetext{
${ }^{4}$ Speziell das Zwei-Grad-Ziel als Grenze für die Erderwärmung. Siehe: Statistisches Bundesamt, Nachhaltige Entwicklung in Deutschland, Indikatorenbericht 2012, Wiesbaden 2012, S. 10 f.

${ }^{5}$ A. Reichel, Das Ende des Wirtschaftswachstums, wie wir es kennen. In: Ökologisches Wirtschaften Heft 1/2013, S. 16.

${ }^{6}$ Ein Indiz in diese Richtung ist die aktuell feststellbare wachsende Anzahl von Publikationen zum Nachhaltigkeitscontrolling. (Vgl. J. Weber, J. Georg, R. Janke, S. Mack: Nachhaltigkeit und Controlling, Weinheim 2012).
}

1.5 Nachhaltiges Wirtschaften wird in erster Linie als technologische Herausforderung verstanden

Nachhaltiges Wirtschaften in Unternehmen ist überwiegend prozessorientiert (fixiert auf ingenieurstechnische Lösungen). Dagegen werden neue Business-Modelle insbesondere im Bereich der Produkte/Dienstleistungen vernachlässigt (DesignThinking von der Kunden- und Wiederverwendungsperspektive beginnend).

Effizienter Umgang mit den begrenzten Ressourcen ist vor allem unter ökonomischen Gesichtspunkten eine sinnvolle Strategie und hat auch ökologisch positive Effekte, wenn sie nicht durch Mengenwachstum überkompensiert wird (Rebound-Effekt). Der Weg in eine nachhaltige Wirtschaftsweise kann nicht in erster Linie durch eine Effizienzstrategie gestaltet werden. Innovationsaktivität darf sich nicht allein auf technologische Lösungen (im Sinne des „GreenTech Made in Germany“) richten. Unternehmen sind aufgefordert, sich auch mit Suffizienzstrategien und sozialen Innovationen (Arbeitsorganisation, Work-Life- Balance partizipative Innovationsstrategien) zu befassen. ${ }^{7}$

Dazu müssten Unternehmen und ihre Verbände auch einen Wandel in der Wissenschafts- und Forschungspolitik unterstützen. Bislang verteidigen sie aber die vorherrschende naturwissenschaftlich-technische Dominanz der Forschungsförderung und erschweren damit sozial-ökologische Forschung, die neue Wohlstandsmodelle (und damit neue Business-Modelle) erkundet - inklusive neuer Dialog-/ Kooperationsformen.

\subsection{Unternehmen thematisieren nachhaltige Lebensstile nicht. Im Gegenteil: Sie fordern zum Konsum auf}

Nachhaltige Lebensstile finden kaum Unterstützung. ${ }^{8}$ Insbesondere Werbung fördert konsumorientierte

\footnotetext{
${ }^{7}$ Vgl. U. Schneidewind, A. Palzkill: Suffizienz als Business Case. Nachhaltiges Ressourcenmanagement als Gegenstand einer transdisziplinären Betriebswirtschaftslehre, Wuppertal 2011.Zur Rolle von Unternehmen für eine Suffizienzpolitik: U. Scheidewind, A. Zahrnt: Damit gutes Leben einfacher wird. Perspektiven einer Suffizienzpolitik, München 2013.

${ }^{8}$ Zur Charakterisierung von Suffizienz hat Wolfgang Sachs die vier E's in die Diskussion gebracht: Entrümpelung: Vereinfachungsstrategien (,Weniger ist mehr"), z. B. Sharing-Modelle, Contracting. Entschleunigung: Langsamer und zuverlässiger handeln. Entkommerzialisierung: Effektivität jenseits des Marktes, Eigenarbeit. Entflechtung: Regionale Wertschöpfung, Dezentralisieren. Vgl. Wolfgang Sachs, Die vier E's: Merkposten für einen maßvollen Wirtschaftsstil. In: Politische Ökologie 11 (33), München 1993.
} 
Lebensstile und muss im Sinne einer nachhaltigen Entwicklung neu ausgerichtet werden. ${ }^{9}$

Während die Bio-Welle rollt, setzen sich die Unternehmen nur wenig mit den strategischen Herausforderungen veränderter Nutzungsmuster und Lebensstile auseinander. Vor allem quantitativ wachstumsorientierte Unternehmen sehen diese Entwicklung als Bedrohung. Sie erkennen nicht, welche Chancen sich ihnen ein ressourcenleichter Lebensstil bietet. Daher versuchen sie mit tradierten Mustern in Marketing und Werbung den Wandel zu einer suffizienzorientierten Lebens-, Arbeits- und Wirtschaftsweise aufzuhalten.

\subsection{Unternehmen unterliegen einer Fixierung auf Wachstum (Wachstumszwang und Wachstumsdrang)}

Unternehmen setzen auf Wachstum und fordern von der Politik Wachstumsimpulse. Überlegungen zu (nachhaltigem) Wirtschaften in einem stagnierenden/ schrumpfenden Umfeld genießen wenig strategische Aufmerksamkeit. Ist Nachhaltiges Wirtschaften ein „Schönwetter“-Thema oder hilft es auch, Unternehmen stabiler zu machen (Stichwort: Resilienz)?

Die Hoffnung, stetiges Wirtschaftswachstum sorge für Wohlfahrt und sozialen Ausgleich, hat sich als trügerisch erwiesen. Die Abkehr vom Wachstumsparadigma ist eine der zentralen Herausforderungen für den politischen und gesellschaftlichen Prozess der Zukunft. Die Transformation in eine nachhaltige Entwicklung kann nur gelingen, wenn Wachstum nicht als der zentrale Imperativ der politischen und wirtschaftlichen Prozesse verstanden wird. Eine ökologisch und sozial ausgerichtete Nachhaltigkeitspolitik muss an die Stelle der überkommenen Wachstumspolitik treten. Die Überwindung der Wachstumsfixierung bedeutet nicht Stagnation, Massenarbeitslosigkeit und Armut oder gar das Ende des gesellschaftlichen Fortschritts. Die Perspektive ändert sich und zwar weg von der eindimensionalen Orientierung auf materielle Güter hin zu nicht-materieller Wohlstandsmehrung und Lebensqualität.

Unternehmen werden sich von der Wachstumsfixierung lösen und sich fragen müssen, wie sie in einem nicht-wachsenden Umfeld prosperieren können. Dies ist mit Resilienz (Widerstandsfähigkeit, Stabilität, Veränderungsfähigkeit) gemeint. ${ }^{10}$ Wenn sie den Prozess einer nachhaltigen Entwicklung unterstützen wollen, dürfen sie sich nicht vom Wachstumsparadigma abhängig machen. Es ist aber festzustellen, dass Unternehmen insbesondere über ihre Verbände

\footnotetext{
${ }^{9}$ Vgl. dazu Corp2020: Accountable Advertising http://www.corp2020. com/accountable-advertising.html/

${ }^{10}$ Vgl. hierzu: I. Seidl, A. Zahrnt, Strukturwandel fordert Neuausrichtung von Geschäftsmodellen. In: Ökologisches Wirtschaften Heft 1/2013, S. 27-29.

und Kammern eine Wachstumspolitik unterstützen und ein wachstumsfreundliches Klima durch niedrige Einkommens-/Körperschaftssteuer und Deregulierung fordern. Die Politik unterstützt diese Strategien durch branchenbezogene Subventionen und eine F\&E-Förderung für hochriskante Technologien (z. B. Atomkraft, Gen- und Nanotechnik), Hilfen für die industrielle Landwirtschaft und den klimaschädlichen fossilen Metabolismus. Vor allem Großunternehmen profitieren von dieser Forschungspolitik. Darüber hinaus leisten Konzerne Widerstand gegen Mit- und Selbstbestimmung bis hin zur Forderung nach unbezahlter Verlängerung der Arbeitszeiten.

1.8 Unternehmen ignorieren weitgehend die internationalen Standards zur Unterstützung einer nachhaltigen Wirtschaftsweise

Deutsche Unternehmen engagieren sich (zu) wenig bei der Formulierung und Anwendung internationaler Standards - von UN Global Compact über EMAS/ ISO bis zu GRI.

Die Wirksamkeit bestehender Methoden und Instrumente, die einen Beitrag zu einer nachhaltigen Wirtschaftsweise leisten können, wird von vielen Unternehmen nicht erkannt bzw. offensichtlich ignoriert.

- EU Eco Management and Audit Scheme (EMAS): Damit lassen sich die Umweltauswirkungen der Produktionsverfahren und Produkte erkennen, dokumentieren und entsprechend den rechtlichen Vorgaben und den eigenen Zielsetzungen gestaltend verändern.

- ISO 26000: Guidance on Corporate Responsibility: Diese Anleitung bietet Orientierung und Unterstützung für die Bewertung der Wirksamkeit gesellschaftlicher Verantwortung.

- Global Reporting Initiative (GRI): Diese internationale Initiative bietet bei der Nachhaltigkeitsberichterstattung hilfreiche Orientierung, mit welchen ökologischen und sozialen Zielen und Indikatoren sich das Unternehmen auseinandersetzen sollte. Die verpflichtende Rechenschaftslegung über ökologische und soziale Einflussfaktoren müsste ebenso selbstverständlich sein wie eine Finanzberichterstattung mit dem Ziel einer umfassenden transparenten und glaubwürdigen Information gegenüber den internen und externen Anspruchsgruppen.

- UN Global Compact: Die Grundsätze umfassen u. a. die aktive Beteiligung von Umwelt- und Menschenrechtsorganisationen z. B. in der Analyse und Verbesserung von globalen Wertschöpfungsprozessen. 
1.9 Unternehmen stellen sich nicht offensiv der Herausforderung zur Umsetzung des Leitbilds einer nachhaltigen Entwicklung

Wenig diskutiert wird die Grundsatzfrage der zukünftigen Rolle von Unternehmen in einer nachhaltigen Gesellschaft. Zwar hat die CSR-Diskussion die extreme Friedman-Position („,The Social Responsibility of Business is to Increase its Profits") deutlich relativiert, jedoch finden Reflektionen und Zukunftsvisionen über Unternehmensziele noch zu wenig Beachtung. Unternehmen sollten in ihrem gesamten Stakeholder-Beziehungsgeflecht Nutzen stiften und Profit als damit verbundene Folgewirkung betrachten.

Über die Abkehr von (kurzfristiger) Rendite-Orientierung ist viel diskutiert worden. Die Idee vom Unternehmen als Institution, die durch Nutzenstiftung für ein Geflecht von Stakeholdern gerechtfertigt ist (,social licence to operate"), wurde bislang nur ansatzweise, vor allem im genossenschaftlichen Kontext, ausgelotet. Eigentümer-Unternehmer haben hier mehr Freiheit zum Experimentieren. Inwieweit solche Experimente zugelassen werden, hängt nicht zuletzt vom Investoren-Verhalten (Banken, Finanzmarkt) ab.

Das Dogma lautet, dass Unternehmen (Personal-)Kosten senken und wachsen müssen, um erfolgreich zu sein. Die Abkehr von diesem Dogma kann unterstützt werden durch die Entwicklung eines empirisch fundierten Leitbilds von (Pionier-)Unternehmen, die erfolgreich wirtschaften, ohne wachsen zu müssen. Durch die Kommunikation eines neuen Leitbilds wird die Dominanz des herrschenden Dogmas gebrochen. ${ }^{11}$

1.10 Ein Ausblick: Es gibt sie doch, die Unternehmen, die nachhaltig wirtschaften. Was aber zeichnet PionierUnternehmen aus?

Nachhaltige Pionier-Unternehmen thematisieren, wie Gewinne erwirtschaftet und wie diese verwendet werden. Sie berichten transparent und stellen sich dem Dialog mit den gesellschaftlichen Anspruchsgruppen, tragen Verantwortung in der Wertschöpfungskette gegenüber ihren Partnern (Lieferanten), Mitarbeiterinnen und Mitarbeitern, dem Staat, der Region.
Auch nachhaltig wirtschaftende Unternehmen erzielen Gewinn. Es stellt sich aber die Frage, welches Geschäftsmodell ein Unternehmen verfolgt, wie Gewinn erwirtschaftet und wie dieser verwendet werden. Entscheidend ist die Wertschöpfung im umfassenden Sinne, nämlich Wert für die Kunden und die Gesellschaft, für diejenigen, die Produkte und Dienstleistungen nutzen und diejenigen, die diese Produkte und Dienstleistungen herstellen. Die Unternehmen übernehmen gesellschaftliche Verantwortung und verändern ihr Kerngeschäft nach Nachhaltigkeitskriterien auch deshalb, weil sie damit die Basis des unternehmerischen Handelns erhalten. Nachhaltige Pionier-Unternehmen erkennen diese Beziehungsstrukturen an und handeln vorsorgend mit Rücksicht auf die natürlichen und sozialen Grundlagen des Wirtschaftens.

\section{Quellen/Literatur/Materialien}

- BUND-Standpunkt „Gesellschaftliche Verantwortung von Unternehmen. Freiwilligkeit und Verpflichtung“, Oktober 2008

- IÖW-Projekt „Postwachstumspioniere - Kommunikationsprojekt zur Erweiterung des Postwachstumsdiskurses um die Rolle mittelständischer Unternehmen“ (http:// www.ioew.de/projekt/Postwachstumspioniere_Kommunikationsprojekt_zur_Erweiterung_des_Postwachstumsdiskurses_um_die_R/)

\footnotetext{
${ }^{11}$ Vgl. A. Zahrnt, I. Seidl, A. Liesen, C. Dietsche: Wachstumsneutrale Unternehmensführung. In: Jahrbuch Ökologie 2014, Stuttgart 2013, S. 141-146.
} 
